\title{
Spin-reorientation transitions in the Cairo pentagonal magnet $\mathrm{Bi}_{4} \mathrm{Fe}_{5} \mathrm{O}_{13} \mathrm{~F}$
}

\author{
Alexander A. Tsirlin,,${ }^{1, *}$ Ioannis Rousochatzakis,,${ }^{2 \dagger}$ Dmitry Filimonov, ${ }^{3}$ Dmitry Batuk, ${ }^{4}$ \\ Matthias Frontzek, ${ }^{5}$ and Artem M. Abakumov ${ }^{3,6, \ddagger}$ \\ ${ }^{1}$ Experimental Physics VI, Center for Electronic Correlations and Magnetism, University of Augsburg, 86159 Augsburg, Germany \\ ${ }^{2}$ School of Physics and Astronomy, University of Minnesota, Minneapolis, Minnesota 55455, USA \\ ${ }^{3}$ Department of Chemistry, Lomonosov Moscow State University, 119991 Moscow, Russia \\ ${ }^{4}$ EMAT, University of Antwerp, Groenenborgerlaan 171, B-2020 Antwerp, Belgium \\ ${ }^{5}$ Quantum Condensed Matter Division, Oak Ridge National Laboratory, Oak Ridge, Tennessee 37831, USA \\ ${ }^{6}$ Skolkovo Institute of Science and Technology, Nobel str. 3, 143026 Moscow, Russia
}

(Received 13 January 2017; revised manuscript received 19 August 2017; published 19 September 2017)

\begin{abstract}
We show that interlayer spins play a dual role in the Cairo pentagonal magnet $\mathrm{Bi}_{4} \mathrm{Fe}_{5} \mathrm{O}_{13} \mathrm{~F}$, on one hand mediating the three-dimensional magnetic order, and on the other driving spin-reorientation transitions both within and between the planes. The corresponding sequence of magnetic orders unraveled by neutron diffraction and Mössbauer spectroscopy features two orthogonal magnetic structures described by opposite local vector chiralities, and an intermediate, partly disordered phase with nearly collinear spins. A similar collinear phase has been predicted theoretically to be stabilized by quantum fluctuations, but $\mathrm{Bi}_{4} \mathrm{Fe}_{5} \mathrm{O}_{13} \mathrm{~F}$ is very far from the relevant parameter regime. While the observed in-plane reorientation cannot be explained by any standard frustration mechanism, our ab initio band-structure calculations reveal strong single-ion anisotropy of the interlayer $\mathrm{Fe}^{3+}$ spins that turns out to be instrumental in controlling the local vector chirality and the associated interlayer order.
\end{abstract}

DOI: 10.1103/PhysRevB.96.094420

\section{INTRODUCTION}

Frustrated magnets [1-3] host a plethora of remarkable collective phenomena, ranging from topological spin liquids, long-range entanglement, and fractionalized excitations [4-10], to emergent electrodynamics and magnetic monopoles [11-13], and even to spin-induced ferroelectricity [14-16]. While the majority of geometrically frustrated magnets are based on spin triangles (or tetrahedra in three dimensions), pentagon-based magnets, which are far more difficult to implement in real materials [17], are now attracting increasing attention both in theory [18-27] and experiment [28-34].

The main interest so far has been in the Cairo pentagonal lattice, a periodic arrangement of irregular pentagons with two types of sites, one with threefold and the other with fourfold connectivity (Fig. 1). Cairo-based models host various phases of classical and quantum nature [24], magnetization plateaus [22,24,26,27], and Kosterlitz-Thouless transitions [22]. At present, there are two main realizations of this lattice: $\mathrm{Bi}_{2} \mathrm{Fe}_{4} \mathrm{O}_{9}[28-31,34]$ and $\mathrm{Bi}_{4} \mathrm{Fe}_{5} \mathrm{O}_{13} \mathrm{~F}$ [32]. A similar pentagonal topology can also be identified in the multiferroics $R \mathrm{Mn}_{2} \mathrm{O}_{5}$ ( $R=\mathrm{Bi}$, Y, or rare earth) [35-42] that are renowned for their complex interplay of commensurate and incommensurate magnetic orders with ferroelectricity.

The symmetric version of the Cairo Heisenberg model has two exchange couplings, namely $J_{33}$ and $J_{43}$ [Fig. 1(a)]. It hosts three phases in the classical, large- $S$ limit [24]: a coplanar orthogonal phase [Fig. 1(b)], a collinear ferrimagnet, and a mixed phase in between. Quantum fluctuations convert the latter to a nonmagnetic and possibly spin-nematic phase for $S=1 / 2$. Additionally, they introduce another collinear

\footnotetext{
*altsirlin@gmail.com

†irousoch@umn.edu

${ }^{\ddagger}$ A.Abakumov@skoltech.ru
}

phase for small $J_{43} / J_{33}$ [24]. This phase features collinear antiferromagnetic order on all fourfold sites and on half of the threefold sites, with the remaining half being disordered [Fig. 1(c)].

$\mathrm{Bi}_{2} \mathrm{Fe}_{4} \mathrm{O}_{9}$ and $\mathrm{Bi}_{4} \mathrm{Fe}_{5} \mathrm{O}_{13} \mathrm{~F}$ feature dumbbells of the fourfold sites. Two Fe1 atoms that comprise a dumbbell lie above and below the $\mathrm{Fe} 2$ plane, centered at a nodal point of the pentagonal lattice. Additionally, there are two inequivalent couplings between fourfold and threefold sites, denoted by $J_{43}$ and $J_{43}^{\prime}$ (Fig. 1), but the classical phase diagram of this extended model is qualitatively the same (Fig. 7). Furthermore, the calculated interactions (reported here and in [25]) place the two compounds nearly on the same spot in the phase diagram, and deep inside the orthogonal phase. Despite this remarkable similarity, the two Cairo materials show qualitatively different behavior. $\mathrm{Bi}_{2} \mathrm{Fe}_{4} \mathrm{O}_{9}$ orders in the anticipated orthogonal state below $238 \mathrm{~K}$, but $\mathrm{Bi}_{4} \mathrm{Fe}_{5} \mathrm{O}_{13} \mathrm{~F}$, where Cairo planes are interleaved by an additional layer of $\mathrm{Fe} 3$ sites, shows three successive transitions at $T_{N} \simeq 178 \mathrm{~K}, T_{2} \simeq 71 \mathrm{~K}$, and $T_{1} \simeq$ $62 \mathrm{~K}$ [32], with three distinct magnetically ordered states that we refer to as phase I $\left(T<T_{1}\right)$, phase II $\left(T_{1}<T<T_{2}\right)$, and phase III $\left(T_{2}<T<T_{N}\right)$. Phase I is orthogonal [32], whereas the nature of phases II and III is unknown to date.

In the following, we unravel the nature of these phases and elucidate their origin. Our main findings are as follows:

(i) Phases I and III are both orthogonal and macroscopically nonchiral, but with opposite local vector chiralities, as defined in Fig. 1(b). Since the two states are degenerate at the level of the isotropic Heisenberg model, anisotropy must play a role.

(ii) The intermediate phase II features nearly collinear spins and drastically reduced moments on half of the $\mathrm{Fe} 2$ sites, reminiscent of the quantum collinear phase of [24]. This is unexpected given the large, "classical" spin $S=5 / 2$ and the fact that we are far from the relevant corner of the phase diagram (Fig. 7). 

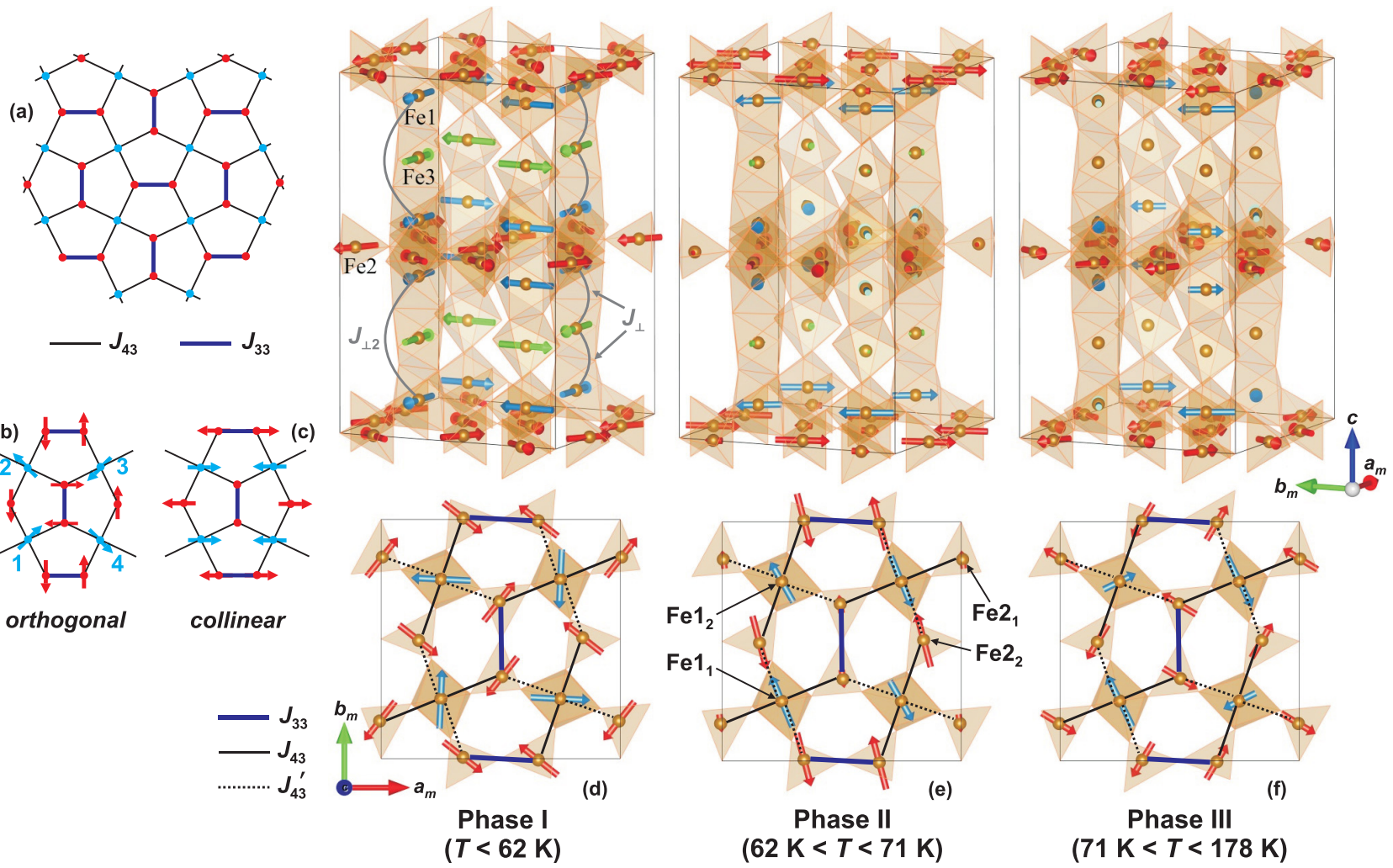

FIG. 1. (a) The symmetric Cairo lattice with two exchange couplings, $J_{33}$ and $J_{43}$. (b),(c) Orthogonal and collinear phases. Note the zero moment on half of the threefold sites in (c). The Fe1 sites 1-4 in (b) provide a measure of local vector chirality as $\chi=\langle\boldsymbol{\Gamma}\rangle /|\langle\boldsymbol{\Gamma}\rangle|$, where $\boldsymbol{\Gamma}=\mathbf{S}_{1} \times \mathbf{S}_{2}+\mathbf{S}_{2} \times \mathbf{S}_{3}+\mathbf{S}_{3} \times \mathbf{S}_{4}+\mathbf{S}_{4} \times \mathbf{S}_{1}$. Note that $\chi$ is defined on the plaquette centered by the $\overline{4}$ rotoinversion axis (and vertical Fe $2_{1}$ dimers). The adjacent plaquettes have opposite chirality, but they are centered by the $4_{2}$ screw axis (and horizontal Fe $2_{2}$ dimers) and thus distinguished by symmetry. The overall magnetic structure is nonchiral. (d)-(f) Magnetic structures of $\mathrm{Bi}_{4} \mathrm{Fe}_{5} \mathrm{O}_{13} \mathrm{~F}$ in phases I, II, and III, respectively. The two types of $J_{43}$ couplings are also indicated. The crystal and magnetic structures are visualized using VESTA [43].

(iii) The onset of phase II upon cooling coincides with a significant growing of the magnetic moments on the interlayer $\mathrm{Fe} 3$ sites, which sit between the Cairo planes and normally act to mediate the three-dimensional (3D) ordering between the planes, as, e.g., in Ref. [44].

(iv) Spin reorientation within the planes is accompanied by a change of the interlayer order from ferromagnetic (phase I) to antiferromagnetic (phase III).

While these features cannot be explained by any standard frustration mechanism involving purely isotropic (Heisenberg) interactions, our $a b$ initio band-structure calculations reveal sizable single-ion anisotropy on the interlayer $\mathrm{Fe}^{3+}$ spins. The Fe3 spins are absent in $\mathrm{Bi}_{2} \mathrm{Fe}_{4} \mathrm{O}_{9}$ (where neighboring planes couple directly to each other), so the emerging physical picture is that the interlayer spins play a vital role for the order within the planes. Specifically, the III $\rightarrow$ II $\rightarrow$ I transitions can be understood as a reorientation of the nominally preferred orthogonal state, from one orientation (phase III) that satisfies the anisotropy on the in-plane spins to another orientation (phase I) that satisfies the anisotropy on the interlayer Fe3 spins, and in between the system must necessarily go through the quasicollinear phase II. This in-plane spin reorientation is accompanied by a change in the interlayer order that evolves from antiferromagnetic in phase III to ferromagnetic in phase I.

\section{MAGNETIC ORDER}

All measurements were performed on single-phase polycrystalline samples of $\mathrm{Bi}_{4} \mathrm{Fe}_{5} \mathrm{O}_{13} \mathrm{~F}$ prepared previously [32] Neutron diffraction data were collected at the cold neutron powder diffractometer DMC (LNS PSI, Villigen, Switzerland) with a wavelength of $4.5082 \AA$ in the $T$ range of $1.5-200 \mathrm{~K}$ in a He cryostat. The magnetic structures were refined by the Rietveld method using the JANA2006 program [45]. The symmetry analysis of possible magnetic configurations was carried out in ISODISTORT [46].

Phases I-III share the same propagation vector $\mathbf{k}=\left(\frac{1}{2}, \frac{1}{2}, 0\right)$. The analysis of irreducible representations (irreps) for the $P 4_{2} / m b c$ nuclear structure with this propagation vector yields six irreps. Their corresponding magnetic space groups and allowed magnetic moment components for different positions of the Fe atoms are listed in Table I. All these symmetries were tested in the refinement of the $T=1.5 \mathrm{~K}$ magnetic structure. The magnetic moments were found to be strictly confined to the $a b$ plane.

The solution was only possible with the $m M_{5}^{-}$irrep and tetragonal magnetic space group $P_{C} 4_{2} / n$. This magnetic structure can be described within the $\mathbf{a}_{m}=\mathbf{a}-\mathbf{b}, \mathbf{b}_{m}=\mathbf{a}+\mathbf{b}$, and $\mathbf{c}_{m}=\mathbf{c}$ magnetic supercell with five positions (Table II) for the magnetic $\mathrm{Fe}$ atoms shown in Fig. 1. We use polar angles $\varphi$ to define orientations of magnetic moments within 
TABLE I. Irreducible representations of $P 4_{2} / m b c$ for $\mathbf{k}=$ $\left(\frac{1}{2}, \frac{1}{2}, 0\right)$, order-parameter directions (OPD), magnetic space groups (mSG), and allowed magnetic moment components for the $\mathrm{Fe}$ positions (referred to the unit cell of the nuclear structure).

\begin{tabular}{lccccc}
\hline \hline Irrep & OPD & mSG & Fe1 & Fe2 & Fe3 \\
\hline$m M_{1}^{+} M_{4}^{+}$ & $(a, b)$ & $P_{C} c c n$ & $\{00 z\}$ & $\{x y 0\}$ & $\{00 z\}$ \\
$m M_{2}^{+} M_{3}^{+}$ & $(a, b)$ & $P_{C} n n n$ & $\{00 z\}$ & $\{x y 0\}$ & none \\
$m M_{5}^{+}$ & $(a, 0)$ & $P_{C} 4_{2} / m$ & $\{x y 0\}$ & $\{00 z\}$ & $\{x y 0\}$ \\
& $(a, a)$ & $P_{A} m n a$ & $\{x y 0\}$ & $\{00 z\}$ & $\{x x 0\}$ \\
& $(a, b)$ & $P_{a} 2 / m$ & $\{x y 0\}$ & $\{00 z\}$ & $\{x y 0\}$ \\
$m M_{1}^{-} M_{4}^{-}$ & $(a, b)$ & $P_{C} n n m$ & $\{00 z\}$ & $\{00 z\}$ & $\{00 z\}$ \\
$m M_{2}^{-} M_{3}^{-}$ & $(a, b)$ & $P_{C} c c m$ & $\{00 z\}$ & $\{00 z\}$ & none \\
$m M_{5}^{-}$ & $(a, 0)$ & $P_{C} 4_{2} / n$ & $\{x y 0\}$ & $\{x y 0\}$ & $\{x y 0\}$ \\
& $(a, a)$ & $P_{A} n n a$ & $\{x y 0\}$ & $\{x y 0\}$ & $\{x x 0\}$ \\
& $(a, b)$ & $P_{c} 2 / c$ & $\{x y 0\}$ & $\{x y 0\}$ & $\{x y 0\}$ \\
\hline \hline
\end{tabular}

the plane, $m_{a}=\mu \cos \varphi$ and $m_{b}=\mu \sin \varphi$ along the $\mathbf{a}_{m}$ and $\mathbf{b}_{m}$ directions, respectively.

\section{A. Phase I}

At $T=1.5 \mathrm{~K}$, two magnetic structures, $\mathrm{A}$ and $\mathrm{B}$, are possible, showing very similar arrangement of the magnetic moments in the pentagonal layers (Fig. 2). These structures can be transformed into each other by rotating all magnetic moments for about $90^{\circ}$. The main difference between these structures is the orientation of a given $\mathrm{Fe} 1_{1}$ moment approximately along the $\mathbf{a}_{m}$ or $\mathbf{b}_{m}$ direction of the magnetic supercell. Although $\mathbf{a}$ and $\mathbf{b}$ (and thus $\mathbf{a}_{m}$ and $\mathbf{b}_{m}$ ) are equivalent in the tetragonal structure, individual Cairo planes lack tetragonal symmetry. Therefore, anisotropy renders a and b distinguishable locally.

With our earlier HRPT data [32], models A and B produced virtually the same refinement residuals. Model A was reported as the magnetic structure in phase I [32], whereas model B was regrettably overlooked in that study. With the DMC data at hand, we can take advantage of the better resolution and sensitivity, and select model B based on the lower refinement residuals; compare $R_{\text {nucl }}=0.023, R_{\text {mag }}=0.027$, and $R_{P}=$ 0.055 for model A and $R_{\text {nucl }}=0.021, R_{\text {mag }}=0.020$, and $R_{P}=0.050$ for model $\mathrm{B}$. The choice of model $\mathrm{B}$ is further corroborated by the analysis of magnetic anisotropy in Sec. III B.

Phase I [Fig. 1(d)] is an orthogonal state, with spins on the $\mathrm{Fe} 1_{1}$ and $\mathrm{Fe} 1_{2}$ sites as well as on the $\mathrm{Fe} 2_{1}$ and $\mathrm{Fe} 22_{2}$ sites being mutually orthogonal. The interlayer ordering is ferromagnetic,

TABLE II. Fractional coordinates of the Fe atoms in the magnetic supercell.

\begin{tabular}{lccc}
\hline \hline & $x / a$ & $y / b$ & $z / c$ \\
\hline $\mathrm{Fe} 1_{1}$ & $\frac{1}{4}$ & $\frac{1}{4}$ & 0.0783 \\
$\mathrm{Fe}_{2}$ & $\frac{1}{4}$ & $\frac{3}{4}$ & 0.0783 \\
$\mathrm{Fe} 1_{1}$ & 0.0057 & 0.8429 & 0 \\
$\mathrm{Fe} 2$ & 0.3429 & 0.0057 & 0 \\
$\mathrm{Fe} 3$ & $\frac{1}{4}$ & $\frac{1}{4}$ & $\frac{1}{4}$ \\
\hline \hline
\end{tabular}
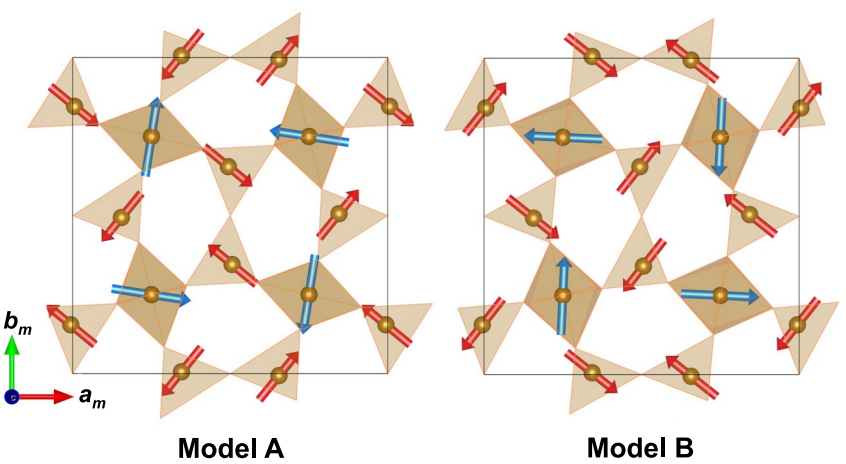

FIG. 2. Distinct magnetic structures A and B at $1.5 \mathrm{~K}$. The Cairo planes at $z=1 / 2$ are shown.

because Fe1 moments of the neighboring Cairo planes interact via $\mathrm{Fe} 3$. The antiferromagnetic alignment of $\mathrm{Fe} 1$ and $\mathrm{Fe} 3$ gives rise to the ferromagnetic arrangement of the $\mathrm{Fe} 1$ moments in the adjacent layers. At $1.5 \mathrm{~K}$, the moments are about $4.0 \mu_{B}$ on the octahedrally coordinated $\mathrm{Fe} 1$ and $\mathrm{Fe} 3$ sites and $3.3 \mu_{B}$ on the tetrahedrally coordinated $\mathrm{Fe} 2$ sites. This difference is due to the stronger $\mathrm{Fe}-\mathrm{O}$ hybridization for the tetrahedrally coordinated $\mathrm{Fe}$ atoms.

\section{B. Phase II}

The propagation vector $\mathbf{k}=\left(\frac{1}{2}, \frac{1}{2}, 0\right)$ is retained in the entire $T=1.5-180 \mathrm{~K}$ temperature range. The refinements were performed assuming that the magnetic structures follow the same irrep and also maintain the order parameter direction and magnetic space group, which would be consistent with the weak first-order nature of the transitions at $T_{1}$ and $T_{2}$ [32]. Satisfactory solutions were indeed found at all temperatures.

As $T$ increases toward $T_{1}$, the $\mathrm{Fe} 1$ and $\mathrm{Fe} 2$ moments remain roughly unchanged, whereas the moment on $\mathrm{Fe} 3$ decreases significantly and drops below $2 \mu_{B}$ at $55 \mathrm{~K}$ (Fig. 3, left). Upon further heating, the magnetic structure changes abruptly entering phase II. All Fe1 sites preserve large moments of $3.2-3.8 \mu_{B}$, whereas the $\mathrm{Fe} 2$ sites split into two groups. The $\mathrm{Fe} 22_{2}$ moments increase to $3.8 \mu_{B}$, whereas the $\mathrm{Fe} 2{ }_{1}$ moments decrease to about $1.2 \mu_{B}$, only one-third of their $1.5 \mathrm{~K}$ value.

Magnetic moments directions change as well. The in-plane magnetic structure of phase II resembles the quantum collinear phase described in Ref. [24]. Deviations from collinearity are due to the fact that $J_{43} \neq J_{43}^{\prime}$. In this case, local fields on the $\mathrm{Fe} 2{ }_{1}$ site do not cancel, leading to the nonzero ordered moment on $\mathrm{Fe} 2{ }_{1}$ and, consequently, to the slight departure of the $\mathrm{Fe} 1_{1}$ and $\mathrm{Fe}_{2}$ moments from the direction of the $\mathrm{Fe} 2_{2}$ moment. Therefore, our phase II can be regarded as an instance of the collinear phase of the Cairo model for the imperfect realization of this model in $\mathrm{Bi}_{4} \mathrm{Fe}_{5} \mathrm{O}_{13} \mathrm{~F}$ [47]. The reasons behind the formation of this phase are rather unusual, though, and will be discussed in Sec. III B.

The second notable change upon the I $\rightarrow$ II transition is the evolution of the interlayer order from ferromagnetic to orthogonal, namely the adjacent Fe1 moments of the two neighboring Cairo planes, which were parallel in phase I, 

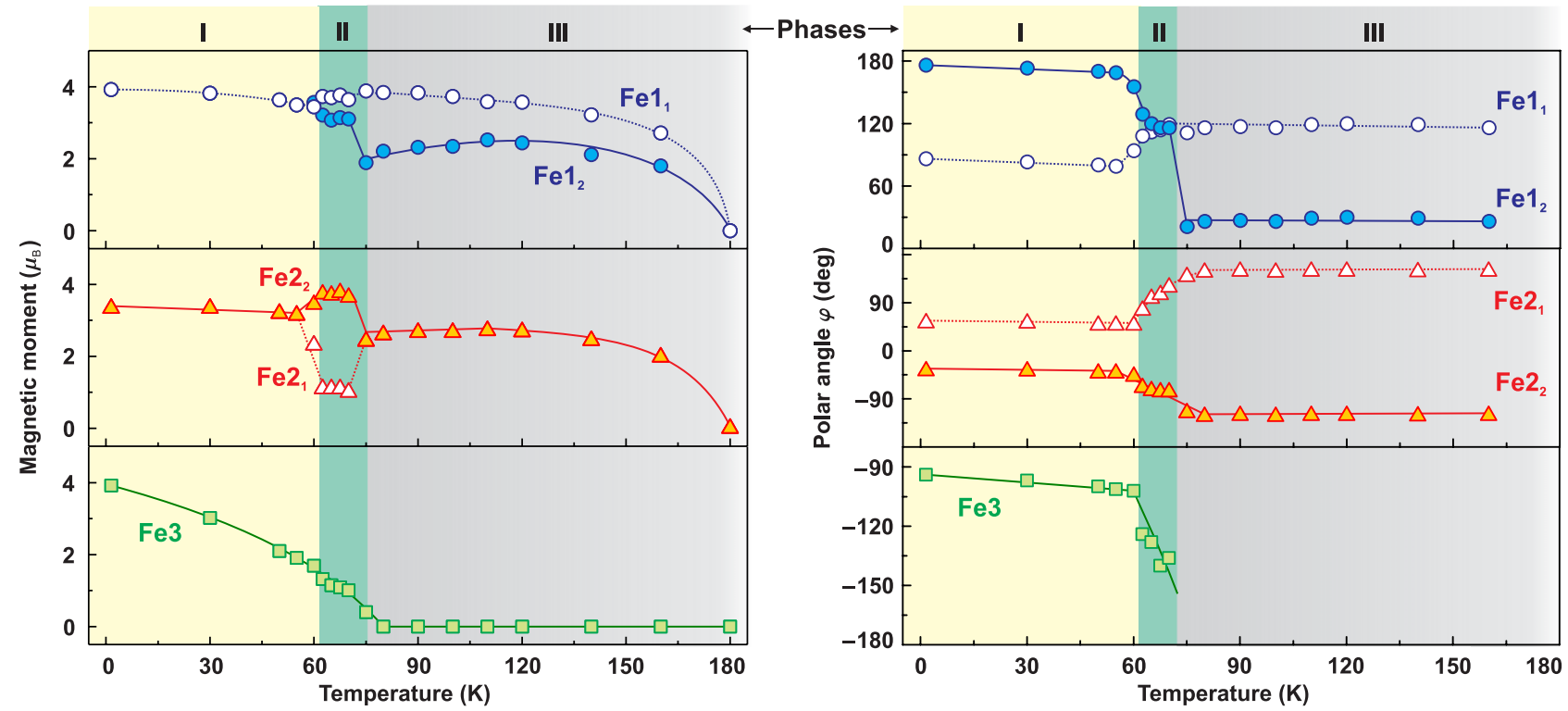

FIG. 3. Left: temperature dependence of the ordered magnetic moments in $\mathrm{Bi}_{4} \mathrm{Fe}_{5} \mathrm{O}_{13} \mathrm{~F}$. Right: temperature dependence of the polar angle $\varphi$ showing abrupt rotations of the moments upon the first-order spin-reorientation transitions at $T_{1}$ and $T_{2}$. The lines are only guides for the eye.

become orthogonal in phase II. This is accompanied by the drastic reduction in the ordered moment on Fe3 (Fig. 3).

\section{Phase III}

The narrow region of phase II is followed by a broader region of phase III, where in-plane order again becomes orthogonal, whereas the interlayer order returns to collinear, but the overall magnetic structure is quite different from that in phase I. First, the interlayer order is now antiferromagnetic, i.e., adjacent $\mathrm{Fe} 1$ moments in the neighboring Cairo planes are antiparallel to each other. Concurrently, the Fe3 moment vanishes. At $100 \mathrm{~K}$ it refined to $0.29(25) \mu_{B}$, which is insignificant given the experimental error bar. Therefore, we fixed the $\mathrm{Fe} 3$ moment to zero throughout the temperature range of phase III.

The differences between the in-plane order of phases I and III can be captured by introducing the local vector chirality $\chi$, which we define for the plaquette of four Fe1 spins as explained in the caption of Fig. 1. The magnetic unit cell contains four such plaquettes. Two of them are centered by the $\overline{4}$ rotoinversion axis, whereas the other two are centered by the $4_{2}$ screw axis. The overall $P_{C} 4_{2} / n$ symmetry requires that adjacent plaquettes have opposite vector chiralities, thus rendering the overall magnetic structure nonchiral. However, each plaquette changes its local vector chirality upon going from phase I to phase III. We find $\chi=+\mathbf{c}$ in phase I and $\chi=-\mathbf{c}$ in phase III for the plaquettes centered by the $\overline{4}$ rotoinversion axis ("vertical $\mathrm{Fe} 22_{2}$ dimers"), and the other way around for the plaquettes centered by the $4_{2}$ axis ("horizontal $\mathrm{Fe} 22_{2}$ dimers").

\section{Mössbauer spectroscopy}

Magnetic structure analysis was supported by Mössbauer spectroscopy measurements. The ${ }^{57} \mathrm{Fe}$ Mössbauer spectra (Fig. 4) were recorded in the temperature range 55-300 K in a transmission mode with a ${ }^{57} \mathrm{Co} / \mathrm{Rh} \gamma$-ray source using a constant acceleration spectrometer MS1104. At room temperature, the spectrum can be decomposed into three doublets with the nearly 40:40:20 ratio of the intensities corresponding to the $\mathrm{Fe} 1, \mathrm{Fe} 2$, and $\mathrm{Fe} 3$ positions, respectively (Table III).

Upon cooling below $T_{N}$, the spectra reveal an additional splitting indicative of the magnetic ordering. However, the spectrum at $100 \mathrm{~K}$, in phase III, could not be accounted for by

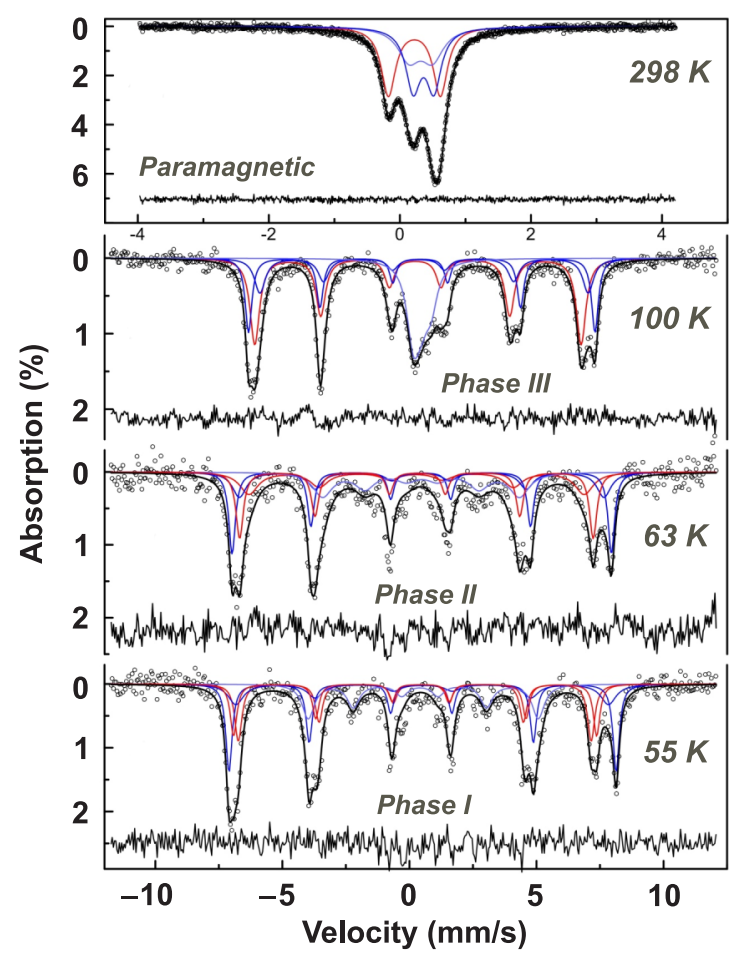

FIG. 4. Mössbauer spectra of $\mathrm{Bi}_{4} \mathrm{Fe}_{5} \mathrm{O}_{13} \mathrm{~F}$ and their fits, as described in the text. For fit parameters, see Table III. 
TABLE III. Parameters of the Mössbauer spectra for $\mathrm{Bi}_{4} \mathrm{Fe}_{5} \mathrm{O}_{13} \mathrm{~F}$. $I$ stands for the fraction of the spectral intensity, $\delta$ is the isomer shift, $\triangle E Q$ is the quadrupolar splitting, $\Gamma$ is the linewidth, and $H$ is the hyperfine field.

\begin{tabular}{|c|c|c|c|c|c|}
\hline & $\begin{array}{c}I \\
(\%)\end{array}$ & $\begin{array}{c}\delta \\
(\mathrm{mm} / \mathrm{s})\end{array}$ & $\begin{array}{c}\Delta E Q \\
(\mathrm{~mm} / \mathrm{s})\end{array}$ & $\begin{array}{c}\Gamma \\
(\mathrm{mm} / \mathrm{s})\end{array}$ & $\begin{array}{c}H \\
(\mathrm{~T})\end{array}$ \\
\hline & & & $T=300 \mathrm{~K}$ & & \\
\hline $\mathrm{Fe} 1$ & 35 & 0.37 & 0.31 & 0.27 & \\
\hline $\mathrm{Fe} 2$ & 38 & 0.23 & 0.78 & 0.26 & \\
\hline \multirow[t]{2}{*}{$\mathrm{Fe} 3$} & 27 & 0.33 & 0.36 & 0.40 & \\
\hline & & & $T=100 \mathrm{~K}$ & & \\
\hline $\mathrm{Fe} 1_{1}$ & 23 & 0.45 & 0.06 & 0.32 & 42.4 \\
\hline $\mathrm{Fe}_{2}$ & 17 & 0.46 & 0.21 & 0.52 & 40.2 \\
\hline $\mathrm{Fe} 2$ & 38 & 0.28 & 0.11 & 0.45 & 39.9 \\
\hline \multirow[t]{2}{*}{$\mathrm{Fe} 3$} & 22 & 0.41 & 0.18 & 0.60 & 22 \\
\hline & & & $T=63 \mathrm{~K}$ & & \\
\hline $\mathrm{Fe} 1_{1}$ & 24 & 0.46 & 0.04 & 0.31 & 46.4 \\
\hline $\mathrm{Fel}_{2}$ & 16 & 0.46 & 0.03 & 0.67 & 44.6 \\
\hline $\mathrm{Fe} 2{ }_{1}$ & 18 & 0.29 & 0.00 & 0.87 & 41.0 \\
\hline $\mathrm{Fe} 2{ }_{2}$ & 22 & 0.29 & -0.04 & 0.35 & 43.2 \\
\hline \multirow[t]{2}{*}{$\mathrm{Fe} 3$} & 21 & 0.47 & -0.05 & 0.93 & 24.1 \\
\hline & & & $T=55 \mathrm{~K}$ & & \\
\hline $\mathrm{Fe} 1_{1}$ & 27 & 0.50 & 0.05 & 0.30 & 47.6 \\
\hline $\mathrm{Fe}_{2}$ & 15 & 0.52 & -0.03 & 0.73 & 45.8 \\
\hline $\mathrm{Fe} 2{ }_{1}$ & 17 & 0.34 & -0.23 & 0.31 & 44.6 \\
\hline $\mathrm{Fe} 22_{2}$ & 20 & 0.34 & -0.26 & 0.34 & 43.4 \\
\hline $\mathrm{Fe} 3$ & 21 & 0.48 & 0.13 & 0.58 & 28.3 \\
\hline
\end{tabular}

a combination of regular sextets. We find that about $20 \%$ of the spectral intensity corresponds to an unresolved sextet with a very weak hyperfine splitting. This signal arises from Fe3 sites that, according to the neutron data, feature a negligible ordered moment above $T_{2}$. Below $T_{2}$, the Fe 3 moments increase and a well-resolved sextet develops.

Below $T_{2}$, the spectrum is decomposed into five sextets (Table III). The reduced ordered moment on $\mathrm{Fe} 22_{1}$ manifests itself in the largely broadened signal. At $55 \mathrm{~K}$, in phase I, the sextets of $\mathrm{Fe} 2{ }_{1}$ and $\mathrm{Fe} 3$ become more narrow, suggesting the formation of large magnetic moments on all Fe sites, which is again in agreement with the magnetic structures shown in Fig. 1.

\section{MAGNETIC MODEL}

\section{A. Isotropic exchange couplings}

The isotropic exchange couplings for $\mathrm{Bi}_{4} \mathrm{Fe}_{5} \mathrm{O}_{13} \mathrm{~F}$ were previously reported in Ref. [32]. However, using these values in numerical simulations of the magnetic susceptibility, we arrived at the too high $T_{N}=250 \mathrm{~K}$ compared to the experimental value of $178 \mathrm{~K}$. Therefore, we revised the microscopic magnetic model using extensive density-functional (DFT) band-structure calculations [48] performed in the FPLO [49] and VASP [50,51] codes. Total energies of collinear spin configurations were mapped onto the spin Hamiltonian, and exchange couplings were determined [52]. The accuracy of this approach was improved by choosing different values of the on-site Coulomb repulsion for the octahedrally coordinated $\mathrm{Fe} 1$ and $\mathrm{Fe} 3$ sites and for the tetrahedrally coordinated Fe2

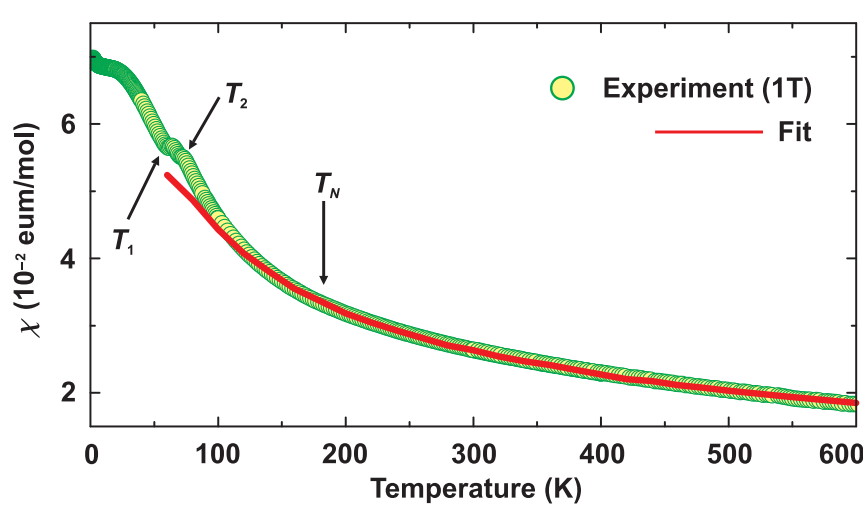

FIG. 5. Fit of the magnetic susceptibility of $\mathrm{Bi}_{4} \mathrm{Fe}_{5} \mathrm{O}_{13} \mathrm{~F}$ [32] using revised exchange parameters reported in this work.

sites, following different oxygen coordination and, therefore, different screening [53].

DFT-based exchange couplings were refined by Monte Carlo simulation of the magnetic susceptibility. The optimized set of exchange parameters was obtained, with $J_{33}=116 \mathrm{~K}$, $J_{43}=38 \mathrm{~K}, J_{43}^{\prime}=57 \mathrm{~K}$, and the $\mathrm{Fe} 1-\mathrm{Fe} 3$ interplane interaction $J_{\perp}=8 \mathrm{~K}$ (Fig. 1). Additionally, a weak second-neighbor interlayer $\mathrm{Fe} 1-\mathrm{Fe} 1$ interaction $J_{\perp 2}=2 \mathrm{~K}$ is present. This set of parameters reproduces the susceptibility down to $120 \mathrm{~K}$ (Fig. 5) and predicts $T_{N} \simeq 180 \mathrm{~K}$, in perfect agreement with the experiment.

\section{B. Magnetic anisotropy}

Several anisotropic terms may occur in $\mathrm{Bi}_{4} \mathrm{Fe}_{5} \mathrm{O}_{13} \mathrm{~F}$. Their calculation follows the same procedure [52], with the only exception being that orthogonal spin configurations are used [53]. Symmetric exchange anisotropy corresponds to energies well below $0.1 \mathrm{~K}$ per $\mathrm{Fe}$ atom and is thus negligible. The antisymmetric exchange anisotropy Dzyaloshinsky-Moriya (DM) interactions allowed on the $J_{43}, J_{43}^{\prime}$, and $J_{\perp}$ bonds are stronger, up to about $5 \mathrm{~K}$ per $\mathrm{Fe}$ atom, but their effect on the magnetic structure largely cancels out, because in the orthogonal structures of phases I and III, the adjacent $J_{43}$ $\left(J_{43}^{\prime}\right)$ bonds feature the same directions of the DM vectors, yet opposite spin rotations.

Single-ion anisotropy terms are believed to be small in $\mathrm{Fe}^{3+}$ compounds due to the $d^{5}$ nature of the magnetic ion. Unexpectedly, we find that these terms are in fact nonnegligible and central to the physics of $\mathrm{Bi}_{4} \mathrm{Fe}_{5} \mathrm{O}_{13} \mathrm{~F}$. Single-ion anisotropy is obtained by fixing spins along a given direction and rotating the reference spin in the plane perpendicular to this direction [53]. Angular dependence of the energy, $E(\varphi)$, directly measures the single-ion anisotropy of the reference spin.

Figure 6 shows single-ion anisotropies for different Fe sites. The single-ion anisotropy of $\mathrm{Fe} 3$ is much stronger than that of $\mathrm{Fe} 1$ and $\mathrm{Fe} 2$. This can be attributed to a large distortion of the $\mathrm{Fe}_{3} \mathrm{O}_{6}$ octahedra [32]. The positions of the energy minima are compatible with the symmetry of the crystal structure, where mirror planes require that the $E(\varphi)$ curves are symmetric with respect to $\varphi=45^{\circ}$ and $135^{\circ}$. 


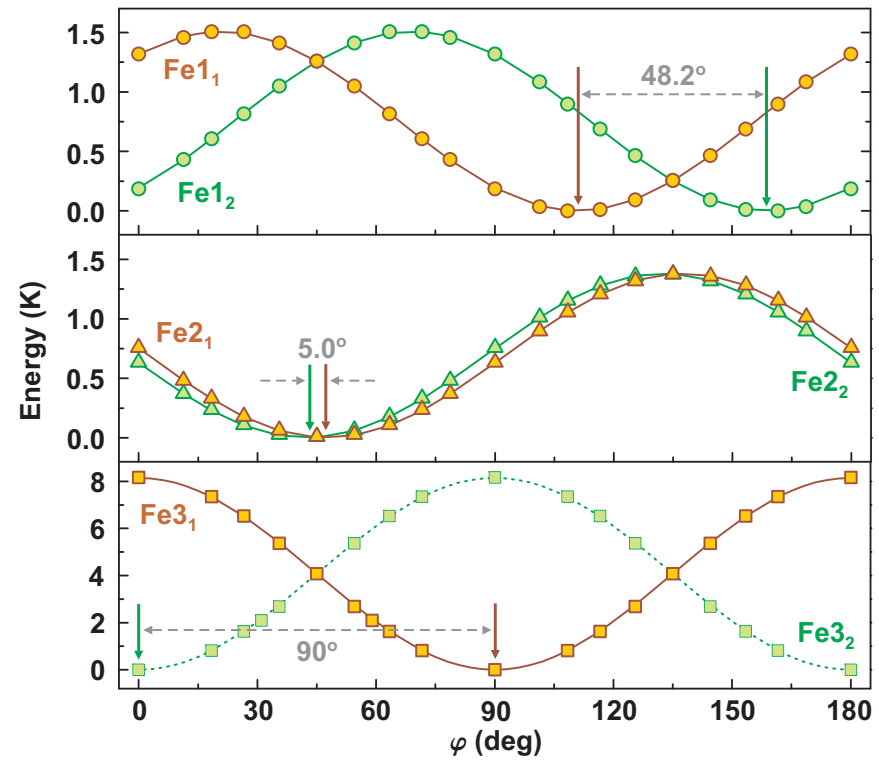

FIG. 6. In-plane single-ion anisotropy energies for the Fe1, Fe2, and $\mathrm{Fe} 3$ sites. The arrows denote preferred spin directions. Only the $\mathrm{Fe} 3$ sites are compatible with the orthogonal structure, because their preferred directions are at $90^{\circ}$ to each other. For the $\mathrm{Fe} 1$ and $\mathrm{Fe} 2$ sites, the angle between preferred directions of the neighboring sites is largely different from $90^{\circ}$. Note that we use $\mathrm{Fe} 3_{1}$ and $\mathrm{Fe} 3_{2}$ for those $\mathrm{Fe} 3$ sites that are coupled to $\mathrm{Fe} 1_{1}$ and $\mathrm{Fe}_{2}$, respectively. The angle $\varphi$ is measured between the magnetic moment and the $\mathbf{a}_{m}$ axis, and all curves are periodic, $E\left(\varphi+180^{\circ}\right)=E(\varphi)$.

An immediate effect of the single-ion anisotropy terms is the selection between states A and B that can form in phase I. Here, the predominant single-ion anisotropy of $\mathrm{Fe} 3$ favors either the $\mathbf{a}_{m}$ or $\mathbf{b}_{m}$ direction of a given $\mathrm{Fe} 3$ atom. The Fe 1 spins choose the same directions because of the isotropic coupling $J_{\perp}$. Therefore, the $\mathrm{Fe} 1_{1}$ spins should point along the $\mathbf{b}_{m}$ axis, whereas the $\mathrm{Fe}_{2}$ spins should point along the $\mathbf{a}_{m}$ axis, as seen in the state $B$ that is pinpointed by our neutron data. The single-ion anisotropy also plays a central role for the selection of both in-plane and interlayer order in $\mathrm{Bi}_{4} \mathrm{Fe}_{5} \mathrm{O}_{13} \mathrm{~F}$, as we explain below.

\section{In-plane order}

The classical phase diagram of the $J_{43}-J_{43}^{\prime}-J_{33}$ model that describes the in-plane isotropic interactions is shown in Fig. 7. This model takes into account the two inequivalent $\mathrm{Fe} 1-\mathrm{Fe} 2$ couplings, $J_{43}$ and $J_{43}^{\prime}$, and the fact that there are two Fe1 spins on each fourfold site of the lattice. The phase diagram has been obtained using Lyons and Kaplan's [54] generalization of the Luttinger-Tisza method [55]; see Ref. [53] for technical details. The phase diagram contains three main phases: the coplanar orthogonal phase, the collinear ferrimagnetic phase, and a mixed phase in between, which is non-coplanar. In the latter phase, the projections of the spins along an axis yield the ferrimagnetic configuration, while the projections in the plane orthogonal to that axis yield the orthogonal configuration; see also Ref. [24]. The relative projections interpolate between 0 and 1 as we go across the two boundaries of this phase.

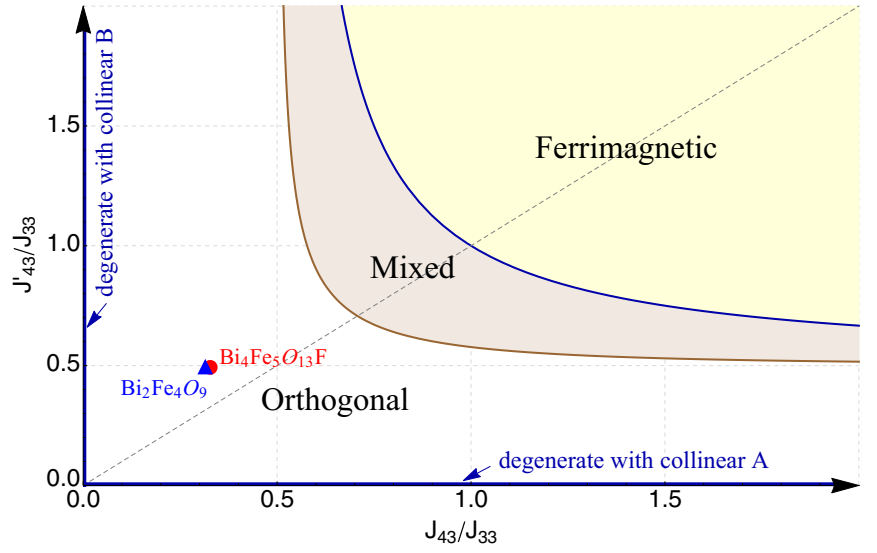

FIG. 7. Classical phase diagram of the isotropic $J_{43}-J_{43}^{\prime}-J_{33}$ model, with two Fe1 spins on each fourfold site of the lattice; see the text. The two available compounds are shown by the filled blue triangle (based on the parameters of [25]) and the filled red dot (based on the parameters given above).

The special lines $J_{43}^{\prime}=0$ and $J_{43}=0$ correspond to decoupled chains. Along these lines, the orthogonal phase becomes degenerate with infinite other ground states, including the so-called collinear phases A and B discussed in [53]. The partially disordered collinear phase of [24], reminiscent of phase II of Fig. 1(e), is stabilized by quantum fluctuations in the corner around $J_{43}=J_{43}^{\prime}=0$. The line $J_{43}=J_{43}^{\prime}$ maps to the model of [24] by rescaling $J_{43} \rightarrow J_{43} / 2$ and $J_{43}^{\prime} \rightarrow J_{43}^{\prime} / 2$.

Based on the above $a b$ initio values, $\mathrm{Bi}_{4} \mathrm{Fe}_{5} \mathrm{O}_{13} \mathrm{~F}$ sits deep inside the orthogonal phase (filled red dot in Fig. 7), and far away from the corner $J_{43}=J_{43}^{\prime}=0$. Interestingly, the second Cairo magnet, $\mathrm{Bi}_{2} \mathrm{Fe}_{4} \mathrm{O}_{9}$, sits almost on the same spot of the phase diagram (filled blue triangle in Fig. 7) according to the ab initio parameters of [25].

The interlayer $\mathrm{Fe} 1-\mathrm{Fe} 3$ coupling is much weaker than the couplings within the plane. Therefore, one expects that the Fe3 spins are more sensitive to thermal fluctuations and decrease much faster than the spins on $\mathrm{Fe} 1$ and $\mathrm{Fe} 2$ [53], in agreement with Fig. 3. On the other hand, the formation of phase II cannot be anticipated, because the system is far away from any collinear phase at zero temperature (Fig. 7). There is a large energy barrier against any thermally driven stabilization of collinearity at the level of the isotropic model. The scenario of quantum fluctuations driving the collinear phase is unlikely as well. The onset of the collinear phase is roughly taking place when the spin length correction $\delta S$ from quadratic spin waves approaches the full value $S=5 / 2$. According to Fig. 4 of Ref. [24], the collinear phase for $S=5 / 2$ (if any) onsets way below $J_{43} / J_{33}=0.1$, and this number should be further divided by 2 , because here we have two Fe1 sites at each fourfold site. So the formation of phase II requires the presence of anisotropy.

The anisotropy of $\mathrm{Fe} 3$ is more than five times stronger than that of $\mathrm{Fe} 1$ and $\mathrm{Fe} 2$. Therefore, at low temperatures, in phase I, Fe 3 with its preferred directions at $\varphi=0^{\circ}$ and $90^{\circ}$ puts the Fe1 moments along $\mathbf{a}_{m}$ and $\mathbf{b}_{m}$. It does not choose the flavor of vector chiral order per se, but the anisotropy of Fe 2 selects $\chi=+\mathbf{c}$ in phase $I$, as confirmed by a direct energy minimization. 

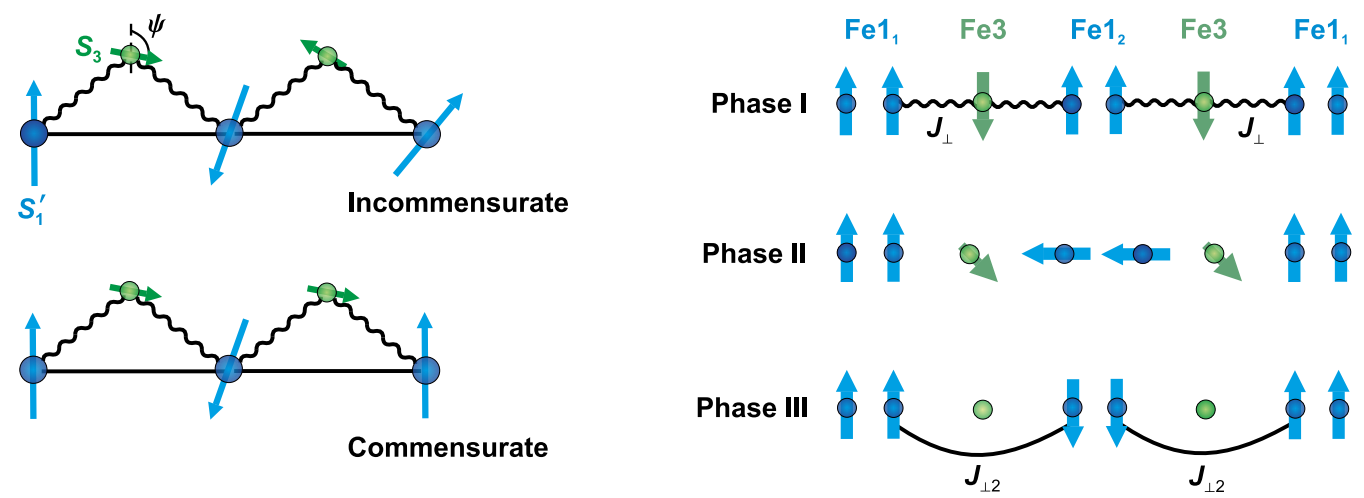

FIG. 8. Left panel: sawtooth-chain model with the couplings $J_{\perp}$ and $J_{\perp 2}$ and two ordered states, incommensurate and commensurate, which are degenerate on the classical level. Right panel: the interlayer order in $\mathrm{Bi}_{4} \mathrm{Fe}_{5} \mathrm{O}_{13} \mathrm{~F}$.

Above $T_{2}$, in phase III, the preferred direction of Fe3 plays no role, and the $\mathrm{Fe} 1$ and $\mathrm{Fe} 2$ moments are left to form an orthogonal configuration, even though their preferred directions are not compatible with such a structure. For example, the preferred directions of $\mathrm{Fe}_{1}$ and $\mathrm{Fe}_{2}$ differ by $48.2^{\circ}$ only, whereas $\mathrm{Fe} 2_{1}$ and $\mathrm{Fe} 2_{2}$ moments prefer to be nearly collinear. However, deviations from the orthogonal structure cost a lot of exchange energy, because the exchange couplings are at least two orders of magnitude stronger than the anisotropy. A clear fingerprint of this competition between the orthogonal state and individual single-ion anisotropies is the large and unexpected difference in the magnetic moments of $\mathrm{Fe} 1_{1}$ and $\mathrm{Fe} 1_{2}$ in phase III. Indeed, the $\mathrm{Fe} 1_{1}$ moment is larger, because it is close to the preferred direction (the departure from the preferred direction is $\Delta \varphi=5^{\circ}$ at $100 \mathrm{~K}$ ). On the other hand, the moment on $\mathrm{Fe}_{2}$ is far away from its preferred direction $\left(\Delta \varphi=47^{\circ}\right)$ and thus $30 \%$ smaller.

A side effect of these energy considerations is that vector chiral order changes from $\chi=+\mathbf{c}$ in phase $\mathrm{I}$ to $\chi=-\mathbf{c}$ in phase III. The continuous transformation between these two phases necessitates the intermediate quasicollinear phase II that exists in a narrow temperature range only.

\section{Interlayer order}

Let us now turn to the interlayer order that can be described by an effective 1D model of the $-\mathrm{Fe} 1_{1}-\mathrm{Fe} 3-\mathrm{Fe} 1_{2}-\mathrm{Fe} 1_{2}-\mathrm{Fe} 3-\mathrm{Fe} 1_{1}-$ chain. It is essentially a ferrimagnetic chain, where $S_{1}^{\prime}=5$ stands for the Fe1 dumbbell and $S_{3}=\frac{5}{2}$ stands for the Fe3 atom. The nearest-neighbor Fe1$\mathrm{Fe} 3$ coupling $J_{\perp}$ is augmented by the next-nearest-neighbor $\mathrm{Fe} 1-\mathrm{Fe} 1$ coupling $J_{\perp 2}$ resulting in a sawtooth-chain geometry (Fig. 8). Classical energy minimization for such a model gives rise to a noncollinear state with the angle $\psi$ between the neighboring spins given by

$$
\cos \psi=-\frac{J_{\perp} S_{3}}{2 J_{\perp 2} S_{1}^{\prime}} .
$$

Two $\psi$ rotations may be followed by another two $\psi$ rotations or by two $-\psi$ rotations. Therefore, there is infinite classical degeneracy in the $J_{\perp}-J_{\perp 2}$ sawtooth-chain model, because any sequence of pairwise $\psi$ and $-\psi$ rotations can occur. This situation is remarkably similar to kagome francisites [56], where the same physics is observed on a 2D lattice, and the ground state is chosen (already on the classical level) by anisotropic terms in the spin Hamiltonian.

In the case of $\mathrm{Bi}_{4} \mathrm{Fe}_{5} \mathrm{O}_{13} \mathrm{~F}, J_{\perp} / J_{\perp 2} \simeq 4$ and $S_{3} / S_{1}^{\prime} \simeq \frac{1}{2}$ produce ferrimagnetic order along the $c$ direction at low temperatures (phase I, Fig. 8). At higher temperatures, $S_{3}$ decreases, and the $S_{3} / S_{1}^{\prime}$ ratio decreases as well. Therefore, $2 \psi$ departs from $360^{\circ}$ and eventually reaches $180^{\circ}$ in phase III, where $S_{3}=0$, and the interlayer order is antiferromagnetic (Fig. 8). Phase II is in the intermediate regime with $2 \psi=270^{\circ}$ (Fig. 8), i.e., the interlayer order is orthogonal with the $90^{\circ}$ configuration between the Fel moments in the adjacent planes.

Ground-state selection requires anisotropic terms in the spin Hamiltonian. The $J_{\perp}-J_{\perp 2}$ sawtooth-chain model makes no difference between the commensurate ("canted") state, where two $\psi$ rotations are followed by two $-\psi$ rotations, and the incommensurate (helical) state, where only $\psi$ rotations occur (Fig. 8, left). This degeneracy is lifted by anisotropy terms.

In $\mathrm{Bi}_{4} \mathrm{Fe}_{5} \mathrm{O}_{13} \mathrm{~F}$, single-ion anisotropy of $\mathrm{Fe} 1$ and $\mathrm{Fe} 3$ is at play. This anisotropy favors the same spin directions on all $\mathrm{Fe} 3$ atoms and, respectively, on all $\mathrm{Fe} 1$ atoms from every second Cairo plane. Therefore, the commensurate order along the c direction is stabilized. It is worth noting that the DM coupling on the $J_{\perp}$ bonds would have an opposite effect and favor the incommensurate helical order, but such a coupling is smaller than the single-ion anisotropy terms providing the energy of $0.4 \mathrm{~K}$ per Fe atom only [57]. Therefore, the single-ion anisotropy is crucial not only for the in-plane order, but also for the commensurate nature of the order between the Cairo planes.

\section{DISCUSSION AND CONCLUSIONS}

The main picture emerging from the experimental data presented here is that the interlayer $\mathrm{Fe} 3$ spins in $\mathrm{Bi}_{4} \mathrm{Fe}_{5} \mathrm{O}_{13} \mathrm{~F}$ play a dual role, on the one hand mediating the $3 \mathrm{D}$ ordering and on the other driving a reorientation of the order both within and between the planes. While details of this transition require further dedicated theoretical work, on the experimental side the effect of the $\mathrm{Fe} 3$ spins is crucial for the design of new Cairo-lattice magnets, because interlayer magnetic sites, which are often introduced for the sake of stabilizing the crystal 
structure [58], are not innocent and in fact play a decisive role for the magnetic order.

The sequence of transitions in $\mathrm{Bi}_{4} \mathrm{Fe}_{5} \mathrm{O}_{13} \mathrm{~F}$ is reminiscent of the consecutive spin re-orientations in $R \mathrm{Mn}_{2} \mathrm{O}_{5}$, where an intermediate collinear phase separates two noncollinear states. However, this collinear phase [37] is different from our phase II, because it does not show the characteristic reduction in the ordered moment on half of the Fe 2 sites. Instead, it may be related to the collinear phases A and B of Fig. 7.

More generally, we show that magnetic order in the pentagonal geometry is largely influenced by even weak anisotropy terms. Despite structural similarities, different systems may eventually show very different types of magnetic order depending on the transition-metal ion. In the case of $\mathrm{RMn}_{2} \mathrm{O}_{5}$, the $d^{4} \mathrm{Mn}^{3+}$ ion is known to be more anisotropic than the $d^{5} \mathrm{Fe}^{3+}$ ion [59], and no direct analogies between Fe-based Cairo magnets and multiferroic $\mathrm{RMn}_{2} \mathrm{O}_{5}$ manganites may occur.

Phase II of $\mathrm{Bi}_{4} \mathrm{Fe}_{5} \mathrm{O}_{13} \mathrm{~F}$ is quite unusual on its own. On the one hand, it strongly resembles the quantum collinear phase of the Cairo model [56]. As explained in Sec. II B, deviations from the collinearity in this phase are due to the imperfect nature of the Cairo lattice $\left(J_{43} \neq J_{43}^{\prime}\right)$. On the other hand, phase II in $\mathrm{Bi}_{4} \mathrm{Fe}_{5} \mathrm{O}_{13} \mathrm{~F}$ is not stabilized by quantum fluctuations, and it originates from competing single-ion anisotropies. Despite this different origin, phase II involves a significant amount of fluctuations reflected in the low ordered moment on $\mathrm{Fe} 22_{1}$. It would be interesting to explore whether properties of phase II and especially its magnetic excitations are similar to those of the quantum collinear phase established in Ref. [56]. Moreover, the variable magnetic structures of $\mathrm{Bi}_{4} \mathrm{Fe}_{5} \mathrm{O}_{13} \mathrm{~F}$ may have an effect on its hitherto unknown dielectric behavior.

\section{ACKNOWLEDGMENTS}

We are grateful to J.-M. Perez-Mato and Dmitry Khalyavin for valuable discussions on the magnetic structures and symmetries. D.F. and A.A. are grateful to the Russian Science Foundation (Grant No. 14-13-00680) for support. A.T. was supported by the Federal Ministry for Education and Research through the Sofja Kovalevskaya Award of the Alexander von Humboldt Foundation. This work is based on experiments performed at the Swiss spallation neutron source SINQ, Paul Scherrer Institut, Villigen, Switzerland.
[1] Introduction to Frustrated Magnetism: Materials, Experiments, Theory, Springer Series in Solid-State Sciences, edited by C. Lacroix, P. Mendels, and F. Mila (Springer, Berlin, 2011).

[2] Frustrated Spin Systems, edited by H. T. Diep, 2nd ed. (World Scientific, Singapore, 2013).

[3] L. Balents, Spin liquids in frustrated magnets, Nature (London) 464, 199 (2010).

[4] P. W. Anderson, Resonating valence bonds: A new kind of insulator?, Mater. Res. Bull. 8, 153 (1973).

[5] S. Yan, D. A. Huse, and S. R. White, Spin-liquid ground state of the $s=1 / 2$ kagome Heisenberg antiferromagnet, Science 332, 1173 (2011).

[6] S. Depenbrock, I. P. McCulloch, and U. Schollwöck, Nature of the Spin-Liquid Ground State of the $S=1 / 2$ Heisenberg Model on the Kagome Lattice, Phys. Rev. Lett. 109, 067201 (2012).

[7] T.-H. Han, J. S. Helton, S. Chu, D. G. Nocera, J. A. RodriguezRivera, C. Broholm, and Y. S. Lee, Fractionalized excitations in the spin-liquid state of a kagome-lattice antiferromagnet, Nature (London) 492, 406 (2012).

[8] A. Kitaev, Anyons in an exactly solved model and beyond, Ann. Phys. (NY) 321, 2 (2006).

[9] L. Savary and L. Balents, Quantum spin liquids: A review, Rep. Prog. Phys. 80, 016502 (2017).

[10] M. J. P. Gingras and P. A. McClarty, Quantum spin ice: A search for gapless quantum spin liquids in pyrochlore magnets, Rep. Prog. Phys. 77, 056501 (2014).

[11] C. Castelnovo, R. Moessner, and S. L. Sondhi, Magnetic monopoles in spin ice, Nature (London) 451, 42 (2008).

[12] C. L. Henley, The Coulomb phase in frustrated systems, Annu. Rev. Condens. Matter Phys. 1, 179 (2010).

[13] J. Rehn and R. Moessner, Maxwell electromagnetism as an emergent phenomenon in condensed matter, Philos. Trans. R. Soc. London, Ser. A 374, 20160093 (2016).

[14] S.-W. Cheong and M. Mostovoy, Multiferroics: A magnetic twist for ferroelectricity, Nat. Mater. 6, 13 (2007).
[15] T. Arima, Spin-driven ferroelectricity and magneto-electric effects in frustrated magnetic systems, J. Phys. Soc. Jpn. 80, 052001 (2011).

[16] Y. Tokura, S. Seki, and N. Nagaosa, Multiferroics of spin origin, Rep. Prog. Phys. 77, 076501 (2014).

[17] This is mostly because of the well-known incompatibility of the five-fold symmetry with lattice periodicity.

[18] R. Moessner and S. L. Sondhi, Ising models of quantum frustration, Phys. Rev. B 63, 224401 (2001).

[19] V. Urumov, Exact solution of the Ising model on a pentagonal lattice, J. Phys. A 35, 7317 (2002).

[20] K. S. Raman, R. Moessner, and S. L. Sondhi, SU(2)-invariant spin- $\frac{1}{2}$ Hamiltonians with resonating and other valence bond phases, Phys. Rev. B 72, 064413 (2005).

[21] A. Jagannathan, B. Motz, and E. Vedmedenko, Novel properties of frustrated low-dimensional magnets with pentagonal symmetry, Philos. Mag. 91, 2765 (2011).

[22] A. Ralko, Phase diagram of the Cairo pentagonal $X X Z$ spin- $\frac{1}{2}$ magnet under a magnetic field, Phys. Rev. B 84, 184434 (2011).

[23] M. Rojas, O. Rojas, and S. M. de Souza, Frustrated Ising model on the Cairo pentagonal lattice, Phys. Rev. E 86, 051116 (2012).

[24] I. Rousochatzakis, A. M. Läuchli, and R. Moessner, Quantum magnetism on the Cairo pentagonal lattice, Phys. Rev. B 85, 104415 (2012).

[25] Z. V. Pchelkina and S. V. Streltsov, Ab initio investigation of the exchange interactions in $\mathrm{Bi}_{2} \mathrm{Fe}_{4} \mathrm{O}_{9}$ : The Cairo pentagonal lattice compound, Phys. Rev. B 88, 054424 (2013).

[26] H. Nakano, M. Isoda, and T. Sakai, Magnetization process of the $S=1 / 2$ Heisenberg antiferromagnet on the Cairo pentagon lattice, J. Phys. Soc. Jpn. 83, 053702 (2014).

[27] M. Isoda, H. Nakano, and T. Sakai, Frustration-induced magnetic properties of the spin- $1 / 2$ Heisenberg antiferromagnet on the Cairo pentagon lattice, J. Phys. Soc. Jpn. 83, 084710 (2014).

[28] N. Shamir, E. Gurewitz, and H. Shaked, The magnetic structure of $\mathrm{Bi}_{2} \mathrm{Fe}_{4} \mathrm{O}_{9}$-Analysis of neutron diffraction measurements, Acta Crystallogr. A 34, 662 (1978). 
[29] A. K. Singh, S. D. Kaushik, B. Kumar, P. K. Mishra, A. Venimadhav, V. Siruguri, and S. Patnaik, Substantial magnetoelectric coupling near room temperature in $\mathrm{Bi}_{2} \mathrm{Fe}_{4} \mathrm{O}_{9}$, Appl. Phys. Lett. 92, 132910 (2008).

[30] E. Ressouche, V. Simonet, B. Canals, M. Gospodinov, and V. Skumryev, Magnetic Frustration in an Iron-Based Cairo Pentagonal Lattice, Phys. Rev. Lett. 103, 267204 (2009).

[31] M. N. Iliev, A. P. Litvinchuk, V. G. Hadjiev, M. M. Gospodinov, V. Skumryev, and E. Ressouche, Phonon and magnon scattering of antiferromagnetic $\mathrm{Bi}_{2} \mathrm{Fe}_{4} \mathrm{O}_{9}$, Phys. Rev. B 81, 024302 (2010).

[32] A. M. Abakumov, D. Batuk, A. A. Tsirlin, C. Prescher, L. Dubrovinsky, D. V. Sheptyakov, W. Schnelle, J. Hadermann, and G. Van Tendeloo, Frustrated pentagonal Cairo lattice in the non-collinear antiferromagnet $\mathrm{Bi}_{4} \mathrm{Fe}_{5} \mathrm{O}_{13} \mathrm{~F}$, Phys. Rev. B 87, 024423 (2013).

[33] M. G. Rozova, V. V. Grigoriev, I. A. Bobrikov, D. S. Filimonov, K. V. Zakharov, O. S. Volkova, A. N. Vasiliev, E. V. Antipov, A. A. Tsirlin, and A. M. Abakumov, Synthesis, structure and magnetic ordering of the mullite-type $\mathrm{Bi}_{2} \mathrm{Fe}_{4-x} \mathrm{Cr}_{x} \mathrm{O}_{9}$ solid solutions with a frustrated pentagonal Cairo lattice, Dalton Trans. 45, 1192 (2016)

[34] S. R. Mohapatra, A. Swain, C. S. Yadav, S. D. Kaushik, and A. K. Singh, Unequivocal evidence of enhanced magnetodielectric coupling in $\mathrm{Gd}^{3+}$ substituted multiferroic $\mathrm{Bi}_{2} \mathrm{Fe}_{4} \mathrm{O}_{9}$, RSC Adv. 6, 112282 (2016).

[35] K. Saito and K. Kohn, Magnetoelectric effect and lowtemperature phase transitions of $\mathrm{TbMn}_{2} \mathrm{O}_{5}$, J. Phys.: Condens. Matter 7, 2855 (1995).

[36] N. Hur, S. Park, P. A. Sharma, J. S. Ahn, S. Guha, and S-W. Cheong, Electric polarization reversal and memory in a multiferroic material induced by magnetic fields, Nature (London) 429, 392 (2004).

[37] G. R. Blake, L. C. Chapon, P. G. Radaelli, S. Park, N. Hur, $\mathrm{S}-\mathrm{W}$. Cheong, and J. Rodríguez-Carvajal, Spin structure and magnetic frustration in multiferroic $R \mathrm{Mn}_{2} \mathrm{O}_{5}(R=\mathrm{Tb}, \mathrm{Ho}$,Dy), Phys. Rev. B 71, 214402 (2005).

[38] C. Vecchini, L. C. Chapon, P. J. Brown, T. Chatterji, S. Park, S-W. Cheong, and P. G. Radaelli, Commensurate magnetic structures of $R \mathrm{Mn}_{2} \mathrm{O}_{5} \quad(R=\mathrm{Y}, \mathrm{Ho}, \mathrm{Bi})$ determined by single-crystal neutron diffraction, Phys. Rev. B 77, 134434 (2008).

[39] A. B. Harris, A. Aharony, and O. Entin-Wohlman, Order Parameters and Phase Diagram of Multiferroic $R \mathrm{Mn}_{2} \mathrm{O}_{5}$, Phys. Rev. Lett. 100, 217202 (2008).

[40] A. B. Sushkov, M. Mostovoy, R. V. Aguilar, S.-W. Cheong, and H. D. Drew, Electromagnons in multiferroic $\mathrm{RMn}_{2} \mathrm{O}_{5}$ compounds and their microscopic origin, J. Phys.: Condens. Matter 20, 434210 (2008).

[41] J. W. Kim, S. Y. Haam, Y. S. Oh, S. Park, S.-W. Cheong, P. A. Sharma, M. Jaime, N. Harrison, J. H. Han, G.-S. Jeon, P. Coleman, and K. H. Kim, Observation of a multiferroic critical end point, Proc. Natl. Acad. Sci. (USA) 106, 15573 (2009).

[42] K. Cao, G.-C. Guo, D. Vanderbilt, and L. He, First-Principles Modeling of Multiferroic $\mathrm{RMn}_{2} \mathrm{O}_{5}$, Phys. Rev. Lett. 103, 257201 (2009).

[43] K. Momma and F. Izumi, VESTA 3 for three-dimensional visualization of crystal, volumetric and morphology data, J. Appl. Crystallogr. 44, 1272 (2011).
[44] R. Nath, K. M. Ranjith, J. Sichelschmidt, M. Baenitz, Y. Skourski, F. Alet, I. Rousochatzakis, and A. A. Tsirlin, Hindered magnetic order from mixed dimensionalities in $\mathrm{CuP}_{2} \mathrm{O}_{6}$, Phys. Rev. B 89, 014407 (2014).

[45] V. Petřriček, M. Dušek, and L. Palatinus, Crystallographic computing system JANA2006: General features, Z. Kristallogr 229, 345 (2014).

[46] B. J. Campbell, H. T. Stokes, D. E. Tanner, and D. M. Hatch, ISODISPLACE: A web-based tool for exploring structural distortions, J. Appl. Cryst. 39, 607 (2006).

[47] We have also tested a different magnetic structure solution that would impose largely noncollinear spins in the spirit of phase I. Toward that end, the space group $P_{A} n n a$ was used ( $P_{C} c n n$ maintaining the crystal axes of $P_{C} 4_{2} / n$ ). This solution, however, demonstrated an inferior fit of the magnetic reflections ( $R_{\text {mag }}=0.030$ at $T=65 \mathrm{~K}$ ) because spin directions for the $\mathrm{Fe} 3$ atoms were constrained by symmetry. A better fit might be achievable with the $P_{c} 2 / c$ magnetic space group, but it contains 10 independent crystallographic positions for the Fe atoms, which renders the solution intractable.

[48] J. P. Perdew, K. Burke, and M. Ernzerhof, Generalized Gradient Approximation Made Simple, Phys. Rev. Lett. 77, 3865 (1996)

[49] K. Koepernik and H. Eschrig, Full-potential nonorthogonal local-orbital minimum-basis band-structure scheme, Phys. Rev. B 59, 1743 (1999).

[50] G. Kresse and J. Furthmüller, Efficiency of ab-initio total energy calculations for metals and semiconductors using a plane-wave basis set, Comput. Mater. Sci. 6, 15 (1996).

[51] G. Kresse and J. Furthmüller, Efficient iterative schemes for $a b$ initio total-energy calculations using a plane-wave basis set, Phys. Rev. B 54, 11169 (1996).

[52] H. Xiang, C. Lee, H.-J. Koo, X. Gong, and M.-H. Whangbo, Magnetic properties and energy-mapping analysis, Dalton Trans. 42, 823 (2013).

[53] See Supplemental Material at http://link.aps.org/supplemental/ 10.1103/PhysRevB.96.094420 for (i) neutron diffraction patterns and their refinements, (ii) details of electronic structure calculations, (iii) details on the derivation of the phase diagram presented in Fig. 7, and (iv) theoretical evidence for the qualitatively different $T$ dependence of the $\mathrm{Fe} 3$ and $\mathrm{Fe} 1 / \mathrm{Fe} 2$ moments. The supplemental material includes Refs. [60-65].

[54] D. H. Lyons and T. A. Kaplan, Method for determining groundstate spin configurations, Phys. Rev. 120, 1580 (1960); T. A. Kaplan and N. Menyuk, Spin ordering in three-dimensional crystals with strong competing exchange interactions, Philos. Mag. 87, 3711 (2007).

[55] J. M. Luttinger and L. Tisza, Theory of dipole interaction in crystals, Phys. Rev. 70, 954 (1946).

[56] I. Rousochatzakis, J. Richter, R. Zinke, and A. A. Tsirlin, Frustration and Dzyaloshinsky-Moriya anisotropy in the kagome francisites $\mathrm{Cu}_{3} \mathrm{Bi}\left(\mathrm{SeO}_{3}\right)_{2} \mathrm{O}_{2} X(X=\mathrm{Br}, \mathrm{Cl})$, Phys. Rev. B 91, 024416 (2015).

[57] For the sake of comparison with Fig. 6, we provide the overall coupling energy that is not normalized to $S=\frac{5}{2}$.

[58] J. Cumby, R. D. Bayliss, F. J. Berry, and C. Greaves, Synthetic analogs of $\mathrm{Fe}(\mathrm{II})-\mathrm{Fe}(\mathrm{III})$ minerals containing a pentagonal "Cairo" magnetic lattice, Dalton Trans. 45, 11801 (2016)

[59] The typical single-ion anisotropy term for octahedrally coordinated $\mathrm{Mn}^{3+}$ is $2-3 \mathrm{~K}[66,67]$, yielding the energy difference on 
the order of $10 \mathrm{~K}$ upon spin rotation, the energy scale similar to that of Fe3 in Fig. 6.

[60] S. Todo and K. Kato, Cluster Algorithms For General$S$ Quantum Spin Systems, Phys. Rev. Lett. 87, 047203 (2001).

[61] A. F. Albuquerque, F. Alet, P. Corboz, P. Dayal, A. Feiguin, S. Fuchs, L. Gamper, E. Gull, S. Gürtler, A. Honecker, R. Igarashi, M. Körner, A. Kozhevnikov, A. Läuchli, S. R. Manmana, M. Matsumoto, I. P. McCulloch, F. Michel, R. M. Noack, G. Pawłowski, L. Pollet, T. Pruschke, U. Schollwöck, S. Todo, S. Trebst, M. Troyer, P. Werner, and S. Wessel, The ALPS project release 1.3: Open-source software for strongly correlated systems, J. Magn. Magn. Mater. 310, 1187 (2007).

[62] A. A. Tsirlin, O. Janson, and H. Rosner, $\beta-\mathrm{Cu}_{2} \mathrm{~V}_{2} \mathrm{O}_{7}$ : A spin-1/2 honeycomb lattice system, Phys. Rev. B 82, 144416 (2010).
[63] W. C. Koehler, E. O. Wollan, and M. K. Wilkinson, Neutron diffraction study of the magnetic properties of rare-earth-iron perovskites, Phys. Rev. 118, 58 (1960).

[64] R. J. McQueeney, J.-Q. Yan, S. Chang, and J. Ma, Determination of the exchange anisotropy in perovskite antiferromagnets using powder inelastic neutron scattering, Phys. Rev. B 78, 184417 (2008).

[65] Y. Abbas, F. Mostafa, and M. Fayek, Antiferromagnetic structure of barium strontium tetraferrate(III), $\mathrm{BaSrFe}_{4} \mathrm{O}_{8}$, Acta Crystallogr. 39, 1 (1983).

[66] F. Moussa, M. Hennion, J. Rodriguez-Carvajal, H. Moudden, L. Pinsard, and A. Revcolevschi, Spin waves in the antiferromagnet perovskite $\mathrm{LaMnO}_{3}$ : A neutron-scattering study, Phys. Rev. B 54, 15149 (1996).

[67] J. Kida and T. Watanabe, Anisotropy and weak ferromagnetism in linear chain antiferromagnet $\left(\mathrm{NH}_{4}\right)_{2} \mathrm{MnF}_{5}$, J. Phys. Soc. Jpn. 34, 952 (1973). 